\title{
Stroke Imaging Research Roadmap
}

\author{
Carlos Leiva-Salinas, MD $^{\mathrm{a}}$ [Research Fellow], Jason Hom, $\mathrm{MD}^{\mathrm{b}}$ [Research Assistant], Steven \\ Warach, MD $^{\mathrm{C}}$ [Chief of the Section on Stroke Diagnostics and Therapeutics], and Max \\ Wintermark, MDd [Associate Professor of Radiology, Neurology, Neurosurgery and \\ Biomedical Engineering, Chief of Neuroradiology] \\ aDepartment of Radiology, Neuroradiology Division. University of Virginia \\ bDepartment of Radiology, Neuroradiology Section. University of California, San Francisco \\ cNational Institute of Neurological Disorders and Stroke, National Institutes of Health \\ dDepartment of Radiology, University of Virginia
}

\begin{abstract}
Though acute stroke imaging has made significant progress in the last few years, a number of improvements and validation steps are needed to make stroke imaging techniques fully operational and appropriate in daily clinical practice. This review outlines the needs in the stroke imaging field and describes a consortium that was founded to achieve them.
\end{abstract}

\section{Keywords}

Stroke; MRI; CT; DWI; Perfusion; Thrombolysis

\section{Introduction: the need for standardization and validation}

Since intravenous tissue plasminogen activator (iv tPA) was approved in 1996, the number of acute stroke patients receiving this treatment has remained under 5\%, mainly because of delayed presentation to care [1]. A recent study suggested that the time window for tPA administration could be extended from 3 hours to 4.5 hours in certain patients [2]. While this extension might slightly increase the rate of tPA administration, it does not change the fundamental limitation, which lies in the fact that we currently decide on administration of thrombolytic therapy using a generalized, statistical rule regarding time to presentation rather than an individualized, pathophysiological assessment of the "ischemic penumbra" which is the target of tPA treatment. Several studies [3-5] have suggested and validated the concept that the ischemic penumbra can be imaged and quantified, and an optimal therapeutic decision regarding thrombolytic agents can be based on such imaging, within an extended time window of up to 9 hours. Such an approach would not only increase the fraction of acute stroke patients amenable to thrombolytic treatment - possibly up to $40 \%$ -

(C) 2011 Elsevier Inc. All rights reserved

Corresponding author for proof and reprints: Max Wintermark, MD University of Virginia, Department of Radiology, Neuroradiology Division. 1215 Lee Street-New Hospital, 1st Floor, Room 1011. PO Box 800170 Charlottesville, VA 22908 434-243-9312 434-982-5753 (fax) Max.Wintermark@virginia.edu.

Publisher's Disclaimer: This is a PDF file of an unedited manuscript that has been accepted for publication. As a service to our customers we are providing this early version of the manuscript. The manuscript will undergo copyediting, typesetting, and review of the resulting proof before it is published in its final citable form. Please note that during the production process errors may be discovered which could affect the content, and all legal disclaimers that apply to the journal pertain.

The authors have nothing to disclose. 
but might also allow more precise patient section, , thereby improving overall clinical outcomes [6].

However, this concept of penumbral image-guided thrombolytic therapy has not been validated in a large phase III trial yet $[7,8]$ and as a result has not become part of the clinical standard-of-care in tPA treatment decisions. One of the reasons for this is the lack of standardization in penumbral imaging, which can result in different treatment decisions in the same patient when different penumbral imaging methods are applied to the same perfusion imaging dataset [9]. Current methods available for defining the penumbra vary for several reasons. The mathematical algorithms that calculate variables such as cerebral blood volume (CBV), cerebral blood flow (CBF) and mean transit time (MTT) differ. The variables that are used to define the penumbra (for example, MTT versus CBF versus CBV in perfusion-CT imaging) and their corresponding thresholds (for example, the threshold of perfusion/diffusion mismatch in MRI imaging) also differ [10] (Figures 1, 2 and 3).

The differences between the imaging methods that identify the ischemic penumbra are not irreconcilable. Rather, they just need some refining in order to give concordant results. This is one of the goals pursued by the Stroke Imaging Repository (STIR) Consortium [11].

\section{The Stroke Imaging Repository (STIR)}

\section{History}

In September 2007, the National Institute of Health, in conjunction with the American Society of Neuroradiology and the Neuroradiology Education \& Research Foundation, sponsored a research symposium entitled Advanced NeuroImaging for Acute Stroke Treatment. That meeting brought together stroke neurologists, neuroradiologists, emergency physicians and neuroimaging research scientists to discuss the role of advanced neuroimaging in acute stroke treatment. In particular, the goals of the meeting were to discuss unresolved issues regarding: (1) the standardization of perfusion and penumbral imaging techniques, (2) the validation of the accuracy and clinical utility of imaging biomarkers of the ischemic penumbra, and (3) the validation of imaging biomarkers relevant to clinical outcome [12].

One of the recommendations was the creation of an international consortium of investigators - the Stroke Imaging Repository - to combine efforts to promote excellence in stroke care and stroke trial design and more specifically to overcome the issues faced by advanced stroke imaging, as mentioned earlier [12].

This central repository was designed to work in collaboration with two other collaborative groups, VISTA (Virtual International Stroke Trials Archive) and MR Stroke. VISTA is an international collaborative venture that was established to facilitate the planning of randomized clinical trials and collects and gathers datasets from multiple institutions. The VISTA database provides the possibility to access a large volume of patient data on which to perform original analyses that would ultimately aid clinical trial design and development $[13,14]$. MR Stroke is a collaborative effort between leading clinicians and clinical scientists around the world with a common interest in Magnetic Resonance Imaging in Stroke [15]. MR Stroke is intended to facilitate the sharing and exchange of information and ideas, to facilitate access to a range of web based MR imaging tools and resources relevant to stroke, and to establish a forum for the exchange of information relevant to research using MR Imaging in stroke. 


\section{Overall Goals}

The overall purpose of STIR is to create a repository of source MRI and CT images that could be used towards the objectives of standardization and validation of image acquisition, of image analysis, and of clinical research methods for image-based stroke research. More specifically, the consortium was originally established to collect and provide a rich resource of patient datasets in order to contribute meaningful information regarding the goals listed above: (1) the standardization of perfusion and penumbral imaging techniques, (2) the validation of the accuracy and clinical utility of imaging biomarkers of the ischemic penumbra, and (3) the validation of imaging biomarkers relevant to clinical outcomes [16].

\section{Main ongoing tasks}

To achieve these goals, the STIR consortium is focusing its initial efforts and resources on three main tasks [17].

Task 1: Review of the literature-A systematic review of the stroke perfusion literature is needed to assess the different parameters and thresholds that have been used to define infarct core and penumbra in acute stroke patients imaged with CT and/or MR perfusion imaging in prior studies or clinical trials. This systematic review will provide an approximation of the accuracy of each of these methods, and of the modifications required to optimize and standardize these methods.

Task 2: Mathematical models, processing algorithms-This task would involve establishing a list of the key post-processing components typically implemented in perfusion software (motion correction, deconvolution, selection of the arterial input function, types of maps produced, and methods to calculate infarct core and penumbra), describing the types/ values of each of these components in the different perfusion software packages (type of deconvolution, delay-sensitivity, number of arterial input functions, etc) and finally assessing the acceptable deviations for the different perfusion software components.

Ideally, the resultant standard perfusion software would fulfill the following premises: (1) the perfusion post-processing would be completely automated and available either on the scanner console, or on a remote workstation, (2) the perfusion software would generate an automated delineation of the infarct core and penumbra, and produce an automated calculation of their volumes, (3) image processing and generation of the maps and volumes would take less than 5 minutes, and (4) processing would be robust despite the use of slightly different image acquisition protocols and different degrees of image quality and noise.

Task 3: Clinical validation of MRI and CT-The different perfusion software packages available, both academic and commercial ones, should be systematically compared, and their results harmonized. In order to achieve this goal, we will collect a large dataset of cases for each modality (MRI and CT). These cases will be randomly divided into two groups. The first set of cases ("training set") will be analyzed separately from the second set ("validation set"). The training set will be used to compare each software package to the the same gold standard dataset, and to determine the required modifications specific to each software package. After this initial phase of refining, the software packages will be "locked", and the improved version will be used to analyze the validation dataset. The results of this analysis will be collected and interpreted centrally, in an independent fashion, in order to validate the improved, locked version of the different perfusion software packages, and show that they all lead to concordant, standardized results, appropriate to use in a stroke treatment trial. 


\section{Next steps, other needs in the field}

Single institutions contributing to the central repository will create a network that will apply standardized imaging acquisition protocols, allowing the constitution of a large, homogenous dataset [12]. This image dataset will facilitate sample size calculations when planning a trial. In addition, studies to determine the optimal imaging modality and the optimal timing to perform imaging to predict clinical outcomes will be made possible. If shorter follow-up periods are validated to assess outcome, and if imaging is demonstrated as an accurate biomarker for clinical outcome, the feasibility of stroke treatment clinical trials will be greatly enhanced, and their cost will be decreased.

All these steps will facilitate and, hopefully, contribute to a successful phase III clinical trial of stroke thrombolytic therapy in an extended time window. This will allow, in turn, a significantly increased number of acute stroke patients who can benefit from thrombolytic therapy and will reduce the burden associated with stroke in our society.

\section{Individual projects}

Analyses and/or research projects taking advantage of the STIR dataset can be proposed by any STIR member through a written application sent to the Steering Committee.

Current Individual projects or study proposals to the Consortium related to imaging techniques, models, standardization and validation include, among others [11]:

\section{Tissue Infarction Risk Maps Applied to Multicenter Stroke data (project} leader: Ona Wu,PhD from Boston, MA)-This study proposes the application of a voxel-wise approach, to define the infarct core and the potentially salvageable tissue, to a large multicenter acute stroke series for model development and validation. Preliminary results based on single center data have shown that these algorithms can be used for tissue characterization $[18,19]$. Traditionally, the presence of mismatch between diffusionweighted MRI (DWI) and perfusion-weighted MRI (PWI) lesion volumes has been considered as the hallmark of potentially salvageable tissue. The use of the PWI/DWI mismatch, however, has been criticized as being suboptimal, due to lesion heterogeneity in both DWI and PWI that can possibly confound accurate assessment of tissue viability within regions of ischemic tissue [20]. In comparison, voxel-wise methods, such as tissue risk maps, may provide a more sensitive and specific approach for identifying potentially salvageable tissue.

\section{Improved Assessment of Infarct Size on Diffusion Magnetic Resonance Imaging; Use of the Graph-Cut Algorithm (project leader: Peter J. Yim, PhD from New Brunswick, NJ)—This study proposes the use of a novel approach to medical image segmentation, a "graph-cut algorithm", for the estimation of the infarct size from diffusion MRI to overcome the subtle but potential meaningful differences obtained with manual methods [21]. The main hypotheses to be tested are, (1) that this method can reduce inter-observer variability in the segmentation of cerebral infarct size from diffusion- weighted magnetic resonance images, and (2) that infarct size estimated by this algorithm from DWI is predictive of final infarct size as assessed at follow-up imaging.}

\section{Predicting Final Extent of Ischemic Infarction Using Artificial Neural Network Analysis of Multi-Parametric MRI in Patients with Stroke (project leader: Hassan Bagher-Ebadian, PhD from Detroit, MI) - In ischemic stroke, the final size of the ischemic lesion is the most important correlate of clinical functional outcome. This study proposes the application of an artificial neural network, to predict the final infarct volume, to a large dataset of treated acute stroke patients. Preliminary results on stroke patients from a}


single center who did not receive thrombolytic therapy have shown that this approach, using baseline MR images, produced a map that correlated well with the T2 weighted abnormality at 3 months. Since this model was trained to predict tissue fate in the absence of any treatment, its performance in treated patient populations remains to be assessed. The proposed study is intended to apply the trained artificial neural network to treated patients (preferably tPA treated patients), and validate its results in a large patient population.

\section{Conclusion}

The establishment of an Acute Stroke Imaging Research Consortium will facilitate the validation and widespread use of imaging for acute stroke patients' care. Currently, the consortium is creating a central repository of imaging studies and clinical data obtained from acute stroke patients, and developing a standardized image analysis toolbox. Contributions to the central repository from academic institutions is crucial not only to obtain a large and diverse dataset, but also to constitute a broad network of international stroke care centers that will - with the collaboration of the NIH, FDA and industry - form the basis for future acute stroke trials, including, but not limited to, treatment of stroke patients with intravenous thrombolytic therapy in an extended time window. This would result in more acute stroke patients being appropriately treated and in an overall improvement of their outcome, as well as in reduced societal costs related to stroke.

\section{References}

1. Bambauer KZ, Johnston SC, Bambauer DE, et al. Reasons why few patients with acute stroke receive tissue plasminogen activator. Arch Neurol. 2006; 63(5):661-4. [PubMed: 16682535]

2. Hacke W, Kaste M, Bluhmki E, et al. Thrombolysis with alteplase 3 to 4.5 hours after acute ischemic stroke. N Engl J Med. 2008; 359(13):1317-29. [PubMed: 18815396]

3. Albers GW, Thijs VN, Wechsler L, et al. Magnetic resonance imaging profiles predict clinical response to early reperfusion: the diffusion and perfusion imaging evaluation for understanding stroke evolution (DEFUSE) study. Ann Neurol. 2006; 60(5):508-17. [PubMed: 17066483]

4. Furlan AJ, Eyding D, Albers GW, et al. Dose Escalation of Desmoteplase for Acute Ischemic Stroke (DEDAS): evidence of safety and efficacy 3 to 9 hours after stroke onset. Stroke. 2006; 37(5):122731. [PubMed: 16574922]

5. Hacke W, Albers G, Al-Rawi Y, et al. The Desmoteplase in Acute Ischemic Stroke Trial (DIAS): a phase II MRI-based 9-hour window acute stroke thrombolysis trial with intravenous desmoteplase. Stroke. 2005; 36(1):66-73. [PubMed: 15569863]

6. Schellinger PD, Warach S. Therapeutic time window of thrombolytic therapy following stroke. Curr Atheroscler Rep. 2004; 6(4):288-94. [PubMed: 15191703]

7. Davis SM, Donnan GA, Parsons MW, et al. Effects of alteplase beyond $3 \mathrm{~h}$ after stroke in the Echoplanar Imaging Thrombolytic Evaluation Trial (EPITHET): a placebo-controlled randomised trial. Lancet Neurol. 2008; 7(4):299-309. [PubMed: 18296121]

8. Hacke W, Furlan AJ, Al-Rawi Y, et al. Intravenous desmoteplase in patients with acute ischaemic stroke selected by MRI perfusion-diffusion weighted imaging or perfusion CT (DIAS-2): a prospective, randomised, double-blind, placebo-controlled study. Lancet Neurol. 2009; 8(2):14150. [PubMed: 19097942]

9. Leiva-Salinas C, Wintermark M. The Future of Stroke Imaging: What We Need and How to Get to It. AJNR Am.J.Neuroradiol. 2010; 41(suppl1):S152-3.

10. Provenzale JM, Wintermark M. Optimization of perfusion imaging for acute cerebral ischemia: review of recent clinical trials and recommendations for future studies. AJR Am J Roentgenol. 2008; 191(4):1263-70. [PubMed: 18806174]

11. Stroke Imaging Repository Home. [accessed September 27, 2010]. found at https://stir.ninds.nih.gov/html/index.html

12. Wintermark M, Albers GW, Alexandrov AV, et al. Acute stroke imaging research roadmap. AJNR Am J Neuroradiol. 2008; 29(5):e23-30. [PubMed: 18477656] 
13. Ali M, Bath PM, Curram J, et al. The Virtual International Stroke Trials Archive. Stroke. 2007; 38(6):1905-10. [PubMed: 17446430]

14. Virtual International Stroke Trials Archive Home. [accessed September 27, 2010]. found at http://www.mrstroke.com/

15. M.R Stroke Home. [accessed September 27, 2010]. found at http://www.vista.gla.ac.uk/

16. Stroke Imaging Repository Charter. [accessed September 27, 2010]. found at https://stir.ninds.nih.gov/html/charter.html

17. Stroke Imaging Repository Projects. [accessed September 27, 2010]. found at https://stir.ninds.nih.gov/html/projects.html

18. Wu O, Christensen S, Hjort N, et al. Characterizing physiological heterogeneity of infarction risk in acute human ischaemic stroke using MRI. Brain. 2006; 129(Pt 9):2384-93. [PubMed: 16891322]

19. Wu O, Koroshetz WJ, Ostergaard L, et al. Predicting tissue outcome in acute human cerebral ischemia using combined diffusion- and perfusion-weighted MR imaging. Stroke. 2001; 32(4): 933-42. [PubMed: 11283394]

20. Ostergaard L, Sorensen AG, Chesler DA, et al. Combined diffusion-weighted and perfusionweighted flow heterogeneity magnetic resonance imaging in acute stroke. Stroke. 2000; 31(5): 1097-103. [PubMed: 10797171]

21. Ay H, Arsava EM, Vangel M, et al. Interexaminer difference in infarct volume measurements on MRI: a source of variance in stroke research. Stroke. 2008; 39(4):1171-6. [PubMed: 18292377] 


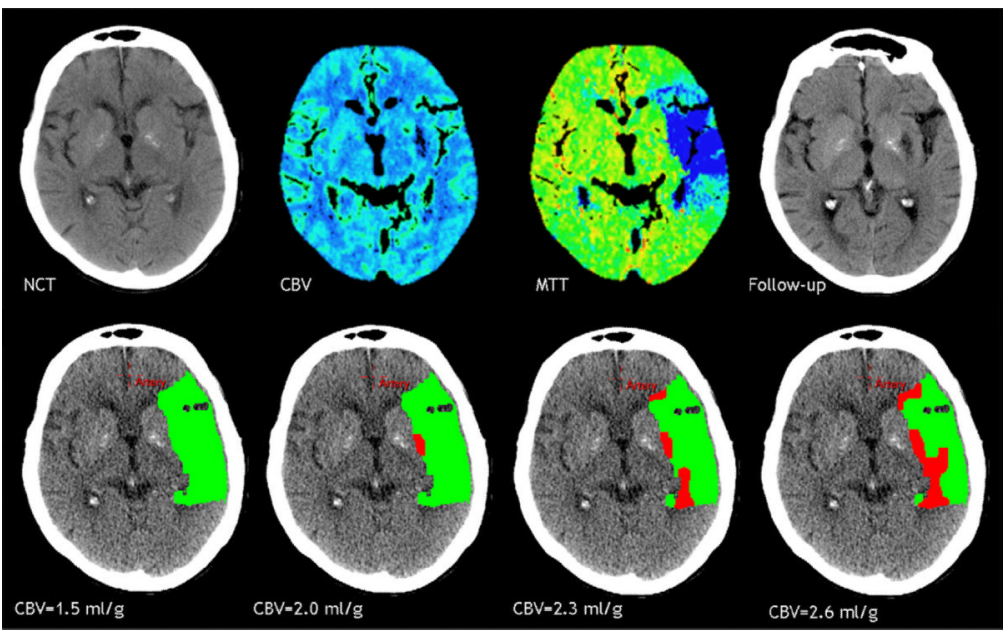

Figure 1.

Depiction of the effect of varying the threshold on the size of the predicted infarct core in an 81 year-old woman with acute left MCA territory infarct treated successfully with tPA. Non contrast CT of the head obtained 2.5 hours after symptom (NCT) onset shows no definite abnormallity. Perfusion CT parametric maps show a mismatch between the region with prolonged MTT (involving the anterior superficial left MCA territory) and the region of decreased CBV (limited to the left putamen), which represents the predicted infarct core. Follow-up non-contrast CT performed 36 hours later shows that the final infarct corresponds to the area of predicted infarct core as represented by the area of decreased CBV. Perfusion CT prognostic maps generated using different CBV thresholds (1.5, 2.0, 2.3 and $2.6 \mathrm{ml} / 100 \mathrm{~g}$ respectively) show that the volume of the predicted infarct core (in red) in case of recanalization varies depending on the chosen value. The CBV value that best predicts the size of the final infarct is $2.0 \mathrm{ml} / 100 \mathrm{~g}$, as published in the literature. The threshold for the delineation of the ischemic penumbra (in green) was set as a relative $145 \%$. 


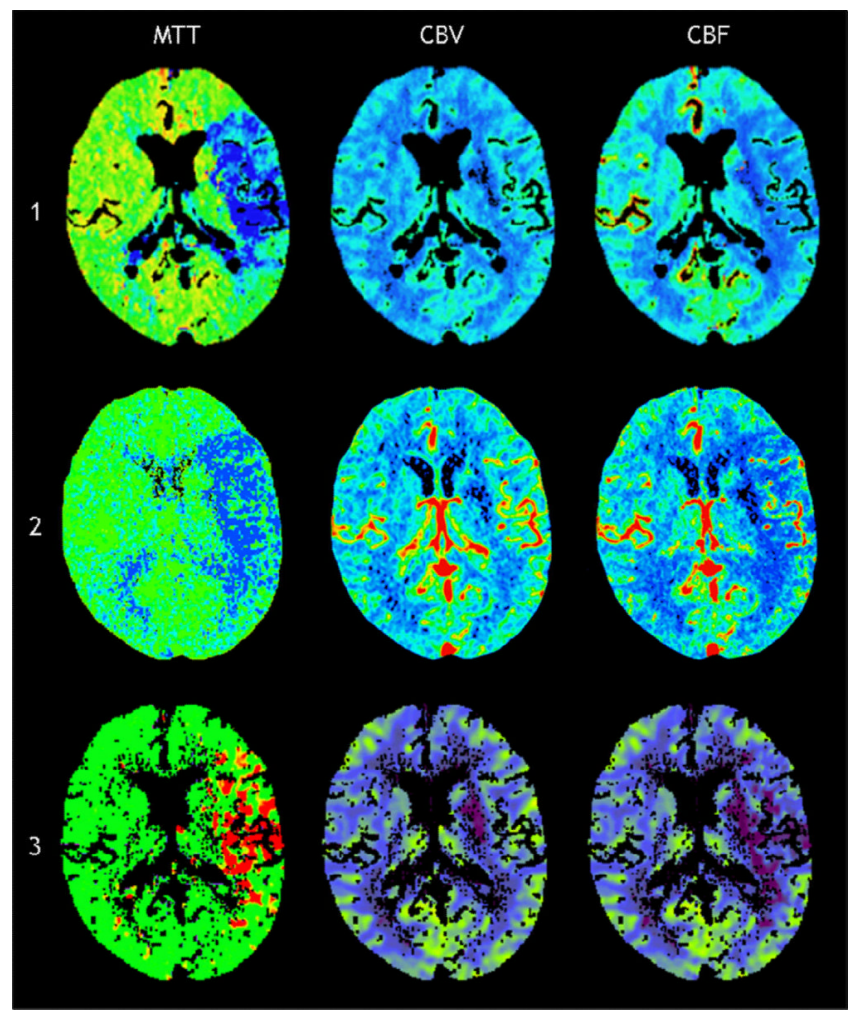

Figure 2.

Depiction of the effect of using different commercial PCT software packages $(1,2$ and 3$)$ on the MTT, CBV and CBF parametric maps in the same patient as in figure 1. Even if the discordance is not major nor is it incongruous, the area of predicted ischemic penumbra as represented by the area of prolonged MTT differs slightly depending on the software employed to process the source images. The area of predicted infarct core as represented by the area of decreased CBV is very similar for all of the 3 manufacturers' tools. Note that parametric maps from manufacturer 1 and 3 are displayed with vessels removed. 


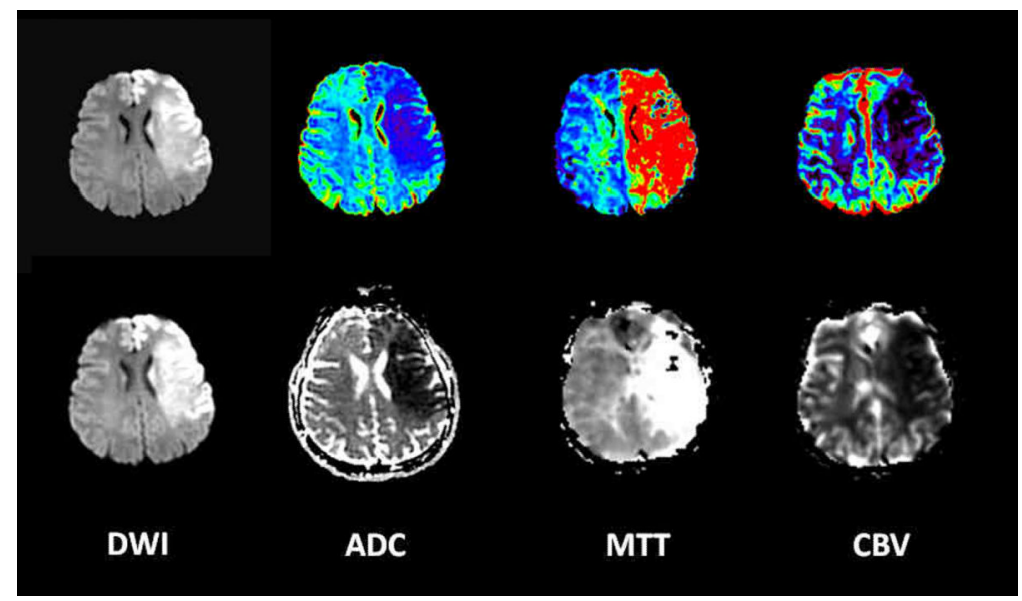

Figure 3.

Comparison of acute diffusion and perfusion maps generated by commercial post-processing software (top row) and by MRI scanner tools (bottom row). Images are from a 59 year old woman one hour and 20 minutes after sudden onset of right hemiparesis and aphasia and a left middle cerebral artery occlusion. The perfusion maps generated from the commercial software were based on an arterial input function model, the scanner generated maps were not. Note that different programs and different algorithms produce differing results for parameters that are nominally the same (e.g., MTT). 